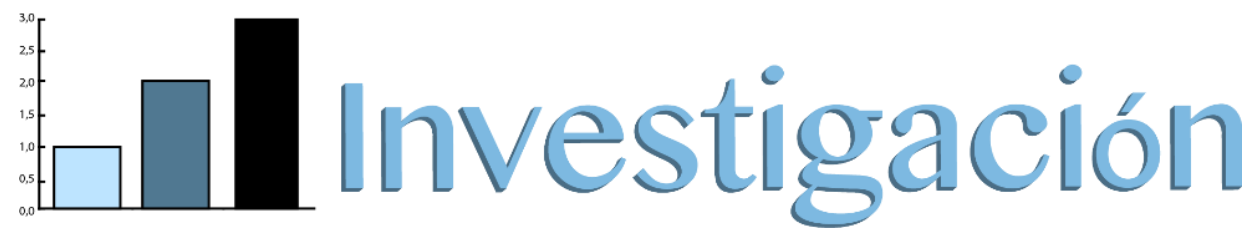

\title{
FACTORES ASOCIADOS A IDEAS SUICIDAS EN ADOLESCENTES ESCOLARIZADOS DE BOGOTA EN 2006, 2013 Y 2018
}

Factors associated with Suicide Ideas in High School Teenagers from Bogotá in 2006, 2013 and, 2018.

Catalina Monroy H., Laura Mogollón Ch., Juan Pablo Molano G., Cristian Leiner Maturana C., Nathalya Oñate N., María Alejandra Ospina C. ${ }^{* *}$ Tutor: Juan Carlos González $\mathrm{Q}^{\dagger+}$ Colaboración: Jenny Pinzón Dibujos: Andrés Reyes $§ \S$

\section{Resumen}

Objetivo: Establecer la relación entre factores psicosociales y el desarrollo de ideas suicidas en adolescentes de un colegio de Bogotá durante los años 2006, 2013 y 2018.

Metodología: Estudio descriptivo de corte transversal. Se aplicó una herramienta a estudiantes adolescentes de un colegio de Bogotá en los años 2006, 2013 y 2018, para indagar por factores sociodemográficos, comportamientos de violencia, funcionalidad familiar, religión, sexualidad, autoestima y consumo de alcohol. Se compararon estas variables con la presencia de ideación suicida.

Resultados: Se encuestaron 2817 estudiantes (1001 en 2006, 475 en 2013 y 1341 en 2018). La baja autoestima (OR 11 IC $95 \%$ $6,9-17,5)$ y la disfunción familiar (OR 15 IC $95 \% 8,4-26,9$ ) representan factores de riesgo importantes para la ideación suicida. La edad con mayor riesgo está en el rango de los 17 a los 20 años.

Palabras clave: Suicidio, violencia, adolescente, autoimagen, familia, factores de riesgo.

\section{Abstract}

Objective: To establish the relationship between psychosocial factors and the development of suicidal ideas in adolescents of a school in Bogotá during the years 2006, 2013 and, 2018.

Methodology: Descriptive cross-sectional study. It was applied a questionnaire to high school teenagers from a school of Bogotá during the years 2006, 2013 and, 2018. The questionnaire inquired about sociodemographic factors, violence behaviors, family functionality, religion, sexuality, self-esteem and, alcohol consumption. These variables were compared with the presence of suicidal ideation.

Results: It were surveyed 2817 students (1001 in 2006, 475 in 2013 and 1341 in 2018). Low self-esteem (OR 11 CI $95 \% 6,9-$ 17,5 ) and family dysfunction (OR 15 CI $95 \% 8,4-26,9$ ) represent important risk factors to develop suicidal ideas. The age of greatest risk is in the range of 17 to 20 years.

Keywords: Suicide, violence, adolescent, self-concept, family, risk factors.

\section{INTRODUCCIÓN}

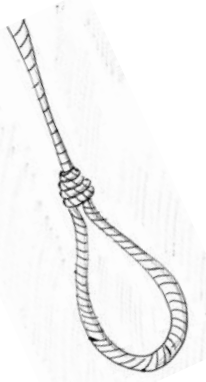

\section{De qué estamos hablando}

La ideación suicida corresponde al conjunto de pensamientos que expresan un deseo o intencionalidad de morir u otras vivencias psicológicas autodestructivas, tales como la fantasía o prefiguración de la propia muerte (1), por lo cual, puede ser considerada como la antesala de la acción consumada. Es documentada por la OMS como segunda causa principal de muerte en el grupo de edad de 15 a 29 años en todo el mundo (2).

** Estudiantes VII semestre 2018 - Facultad de Medicina FUJNC.

+† MD. Especialista Med. Familiar Integral, MSP. Director Departamento de Medicina Comunitaria FUJNCjuan.gonzalez@juanncorpas.edu.co

$\neq \neq$ Coeditora Carta Comunitaria.

$\S \S$ MD. Cirujano General. 


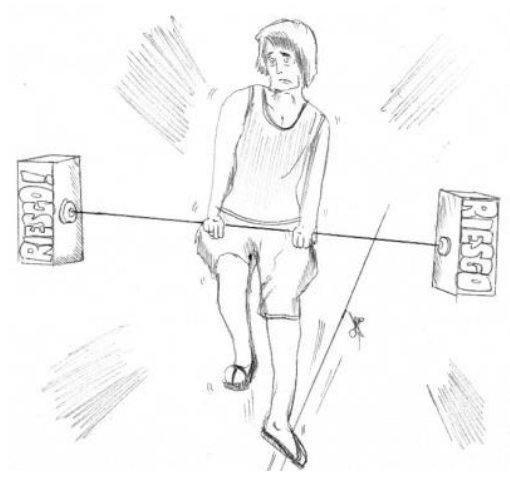

El riesgo de ideación suicida puede verse aumentado en los adolescentes dada su vulnerabilidad ante las posibles situaciones problemáticas que pudiesen enfrentar, tales como las dificultades familiares graves, los comportamientos de violencia o de agresividad, los antecedentes de abuso sexual, y/o, las circunstancias menos dramáticas -aunque, para ellos trascendentales, como, por ejemplo, el obtener bajas calificaciones, por su pobre respuesta ante el estrés y frustración que ello les genera (3). Además, está el riesgo derivado del consumo de alcohol y otras sustancias psicoactivas que hace que tengan más probabilidad de desarrollar una conducta suicida (1).

Tales situaciones resultan ser factores de riesgo importantes si se tiene en cuenta que en la adolescencia también aparecen ideas o procesos de depresión y ansiedad y que, la autoestima, hace su presentación social, siendo de riesgo cuando la autopercepción es negativa (4).

\section{Una diferencia importante}

El suicidio consumado representa un problema de salud pública importante y en Colombia corresponde a la cuarta causa de muerte en sujetos entre los 12 y los 15 años de edad (5). Importante es diferenciar el pensar en el suicidio, que no es infrecuente y de por sí no se percibe como anormal
(2). El riesgo se presenta cuando existe una secuencia de eventos que se dan de manera progresiva y que van desde el pensamiento, seguido de planes suicidas (querer hacerlo) que culminan en uno o múltiples intentos con aumento progresivo hasta llegar a la muerte (suicidio consumado) (1).

\section{Edad y género}

En el mundo aproximadamente 800000 personas se suicidan al año y resulta relevante el hecho de que esta cifra incluye un número significativo de jóvenes, tal como se evidenció durante 2015, cuando el suicidio fue la segunda causa de defunción en el grupo de 15 a 29 años a nivel mundial (2). En los adultos se ha encontrado que las mujeres lo intentan más, pero que los hombres lo consuman más (6).

\section{Autopercepción y baja autoestima}

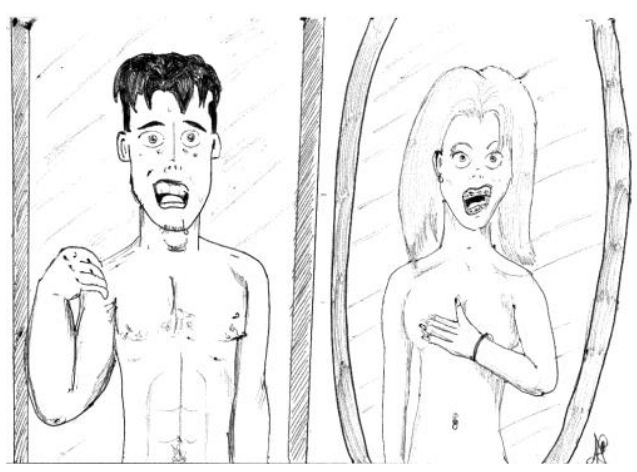

La autoestima se define como autopercepción y valoración de sí mismo con base en la cual las personas se comportan ante los demás (5) y se considera clave en el desarrollo de la salud mental del ser humano. En la adolescencia hace su aparición $y$, por ello, en tanto se afianza, puede fluctuar siendo una condición particularmente importante en el proceso suicida (7). Como viva y reaccione el joven a las situaciones que se le presenten será clave en su consolidación (8), lo cual puede ser interpretado como una crisis normativa propia de la edad. Situaciones que generen respuestas donde se sientan mal pueden dar inicio de ideaciones suicidas como mecanismo inapropiado. Por el contrario, si ante situaciones difíciles, el adolescente las asume, sintiéndose bien (9), la autoestima se afianza y con ello reduce los sentimientos de frustración en torno a sus relaciones, en particular con sus compañeros y su familia. 


\section{Violencia}

La violencia se define como el uso deliberado de la fuerza física o el poder, sea en grado de amenaza o efectivo, contra uno mismo, otra persona o un grupo o comunidad, que cause o tenga muchas probabilidades de causar lesiones, muerte, daños psicológicos, trastornos del desarrollo o privaciones (10). Los jóvenes que han sido víctimas de la violencia son más propensos al suicidio (11) dado que el comportamiento belicoso contra ellos les da la idea de ser sometidos, afectando la confianza en sí mismos y en los demás (12). El bullying es una evidente situación de eso (13), donde, paradojalmente, la víctima se puede convertir en victimario en el futuro. Lo cierto es que el acoso escolar es un macroproblema en salud; se ha calculado que casi 1 de cada 3 adolescentes ha estado expuesto a este acoso (10). En Colombia, de acuerdo con el DANE, al menos el $11 \%$ de los adolescentes han notificado haber sido víctimas en Bogotá de algún tipo de violencia (14). Lo anterior sin dejar de lado la violencia intrafamiliar cuya denuncia está en ascenso (más reportes de casos de maltrato infantil (15).

\section{Consumo de alcohol}

El consumo de alcohol es una situación muy frecuente en los jóvenes suicidas (16). Se ha encontrado que cerca del $45 \%$ de los jóvenes que se suicidaron tenían niveles de alcohol importantes. Además, en jóvenes con problemas de consumo excesivo de alcohol, el suicidio en casi 10 veces más frecuente (17).

\section{La familia}

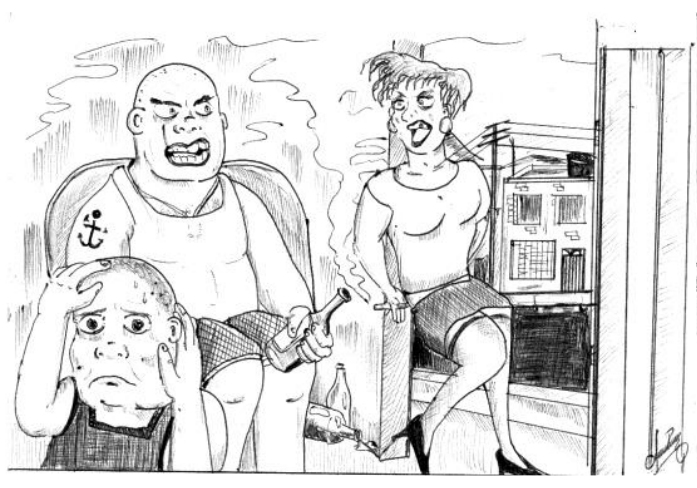

El valor de la familia en la vida de todas las personas es un hecho indiscutible resaltado universalmente (18). El desarrollo psicológico de los jóvenes está poderosamente relacionado con la funcionalidad familiar (19) sea para bien o para mal (20), según sea el caso, compartiendo una

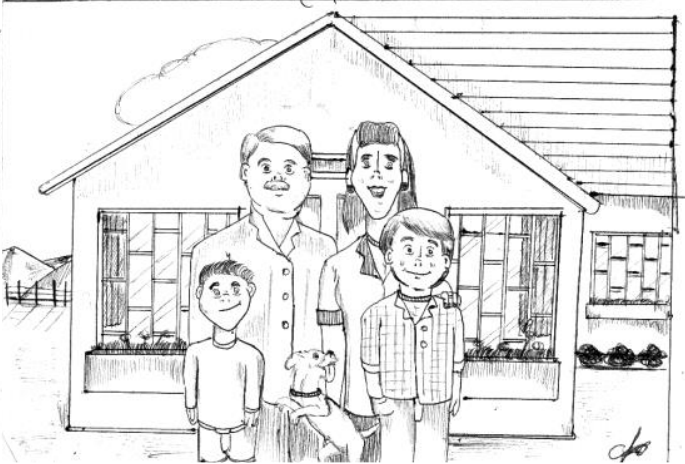

responsabilidad grande en el desarrollo de la salud mental de los individuos, siendo importante resaltar que la disfunción familiar es un factor relacionado con la aparición de síntomas depresivos con importancia clínica $(21,22)$.

\section{Religión}

La religión, entendida como un conjunto de creencias espirituales que determinan ciertos comportamientos (23), acompaña al ser humano y le confiere capacidad para enfrentar situaciones, por lo que puede ser considerada como un factor protector de conductas suicidas (24).

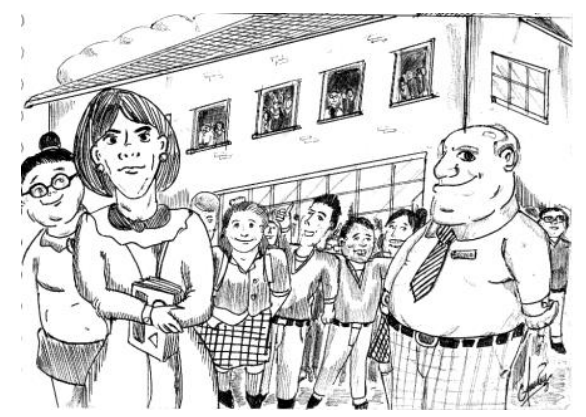

Esta investigación surgió como parte de la respuesta a una psicóloga orientadora de jóvenes de un colegio, que expresó su preocupación por la atención, cada vez más frecuente, a estudiantes que presentan ideas suicidas con intentos fallidos. Dado que se ha estado acompañando a dicho colegio mediante diagnósticos comunitarios, se quiso indagar por los factores psicosociales relacionados con la planeación suicida. Se espera que, con base en los resultados, se puedan proponer intervenciones preventivas en tal sentido. 


\section{MATERIAL Y MÉTODOS}

Tipo de estudio: Estudio descriptivo de corte transversal.

Población: Estudiantes de un colegio público de Bogotá encuestados en los años 2006, 2013 y 2018.

Criterios de inclusión: Estudiantes de bachillerato de un colegio público de Bogotá que participaron voluntariamente, previo consentimiento informado de sus padres.

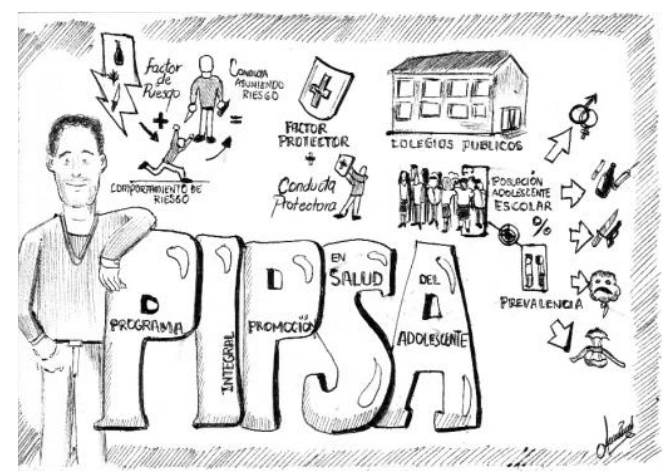

Variables de estudio: El instrumento usado consta de 60 preguntas alusivas a diversas áreas. La variable dependiente fue haber pensado en el suicidio (no lo ha pensado, sí lo ha pensado y le gustaría hacerlo, referido este último a haberlo planeado). Como variables explicativas, se indagaron aspectos sociodemográficos (género, edad y estrato social); percepción del ánimo al momento de participar en el estudio (feliz, normal o triste); funcionalidad familiar (medida a través del test de APGAR original (25), que clasifica la funcionalidad como normal, disfunción moderada o severa); estructura familiar (nuclear completa, incompleta, extensa, reconstituida y sin padres); consumo de alcohol en la última semana, así como la cantidad que bebió; se hicieron tres preguntas del test de Cage (26) (si ha tenido dificultades por la forma de beber, si se ha hecho daño bajo el efecto del alcohol y si siente que necesita consumir más alcohol); percepción que tiene de sí mismo (cree que en el futuro alcanzará lo que quiere, está satisfecho consigo mismo, le gustaría cambiar muchas partes de su cuerpo y siente rechazo de compañeros que le dicen que es torpe); religiosidad (practica su religión y se considera una persona religiosa).

Recolección de la información: Se realizó en tres diferentes momentos (2006, 2013 y 2018), en las aulas del colegio. Se entregó el instrumento impreso a cada uno de los estudiantes que deseaban participar y que contaban con el permiso previo de sus padres; se aclaró el propósito del estudio y su carácter anónimo y voluntario. Se solicitó que contestaran con la mayor veracidad posible. El tiempo máximo para contestar fue de 30 minutos aproximadamente, pudiendo salir del salón quien la contestara antes.

Manejo estadístico: Los datos se tabularon en el programa Excel. Se comparó la variable "Ha pensado en el suicidio y le gustaría hacerlo (lo ha planeado)" versus "No lo ha pensado" con las variables explicativas usando el programa Epi Info con el cual se obtuvieron los OR, los intervalos de confianza y la prueba $p$.

\section{RESULTADOS}

Tabla 1. Caracteriticas demográficas y estado de ánimo de adolescentes de un colegio medidas en tres momentos diferentes (2006, 2013 y 2018).

\begin{tabular}{|c|c|c|c|c|c|c|}
\hline \multirow{2}{*}{$\begin{array}{l}\text { CARACTERÍSTICAS } \\
\text { DE LA POBLACIÓN }\end{array}$} & \multicolumn{2}{|c|}{2006} & \multicolumn{2}{|c|}{2013} & \multicolumn{2}{|c|}{2018} \\
\hline & $\mathbf{N}^{\circ}$ & $\%$ & $\mathbf{N}^{\circ}$ & $\%$ & $\mathbf{N}^{\circ}$ & $\%$ \\
\hline \multicolumn{7}{|l|}{ Edad } \\
\hline De 10 a 12 años & 286 & 29,2 & 171 & 32,1 & 315 & 23,5 \\
\hline De 13 a 16 años & 606 & 61,8 & 316 & 59,4 & 849 & 63,3 \\
\hline De 17 a 20 años & 89 & 9,1 & 45 & 8,5 & 178 & 13,3 \\
\hline \multicolumn{7}{|l|}{ Sexo } \\
\hline Masculino & 459 & 45,9 & 284 & 53,3 & 711 & 53,0 \\
\hline Femenino & 542 & 54,1 & 249 & 46,7 & 630 & 47,0 \\
\hline \multicolumn{7}{|l|}{ Estrato } \\
\hline Uno y dos & 794 & 81,1 & 325 & 63,1 & 608 & 46,9 \\
\hline Tres y cuatro & 178 & 18,2 & 182 & 35,3 & 676 & 52,2 \\
\hline Cinco y seis & 7 & 0,7 & 8 & 1,6 & 12 & 0,9 \\
\hline \multicolumn{7}{|l|}{ Estado de ánimo } \\
\hline Triste & 77 & 7,7 & 29 & 5,5 & 88 & 6,6 \\
\hline Feliz & 293 & 29,4 & 189 & 35,5 & 395 & 29,5 \\
\hline Normal & 627 & 62,9 & 314 & 59,0 & 855 & 63,9 \\
\hline
\end{tabular}


Los jóvenes en la etapa exuberante de la adolescencia (13 a 16 años) son la mayoría en los tres momentos. Se observa un aumento en la percepción de condición económica.

Tabla 2. Estructura, función familiar, consumo de alcohol, percepciones de autoestima, violencia y religiosidad de adolescentes de un colegio en tres momentos diferentes (2006 2013 y 2018).

\begin{tabular}{|c|c|c|c|c|c|c|}
\hline \multirow{2}{*}{ VARIABLE } & \multicolumn{2}{|c|}{2006} & \multicolumn{2}{|c|}{2013} & \multicolumn{2}{|c|}{2018} \\
\hline & $\mathbf{N}^{\circ}$ & $\%$ & $\mathbf{N}^{\circ}$ & $\%$ & No & $\%$ \\
\hline \multicolumn{7}{|l|}{ Funcionalidad familiar } \\
\hline Disfunción severa & 48 & 5,2 & 22 & 4,4 & 77 & 5,8 \\
\hline Disfunción moderada & 230 & 24,8 & 153 & 30,7 & 409 & 30,9 \\
\hline Funcionalidad normal & 650 & 70,0 & 324 & 64,9 & 839 & 63,3 \\
\hline \multicolumn{7}{|l|}{ Estructura familiar } \\
\hline Nuclear (vive con papá, mamá y hermanos) & 556 & 62,6 & 292 & 54,9 & 623 & 47,0 \\
\hline Incompleta (falta uno de los padres) & 238 & 26,8 & 156 & 29,3 & 500 & 37,7 \\
\hline Extensa (padres, hermanos y otros) & 91 & 10,2 & 41 & 7,7 & 113 & 8,5 \\
\hline Reconstituida (padrastro y madrastra) & 3 & 0,3 & 16 & 3,0 & 30 & 2,3 \\
\hline Sin padres & 0 & 0,0 & 27 & 5,1 & 59 & 4,5 \\
\hline \multicolumn{7}{|l|}{ Consumo de alcohol } \\
\hline Ha bebido alcohol en la última semana & 269 & 27,1 & 172 & 32,6 & 417 & 31,4 \\
\hline Solo una vez* & 162 & 16,3 & 108 & 20,5 & 220 & 16,6 \\
\hline Entre dos y tres veces* & 80 & 8,1 & 45 & 8,5 & 140 & 10,6 \\
\hline Entre cuatro y seis veces* & 10 & 1,0 & 11 & 2,1 & 35 & 2,6 \\
\hline Entre 7 y más veces* & 17 & 1,7 & 8 & 1,5 & 22 & 1,7 \\
\hline Ha tenido dificultades por la forma de beber & 45 & 4,6 & 35 & 6,7 & 85 & 6,4 \\
\hline $\begin{array}{l}\text { Se ha hecho daño (o lo ha hecho) bajo efecto del } \\
\text { alcohol }\end{array}$ & 19 & 1,9 & 20 & 3,9 & 27 & 2,0 \\
\hline Necesita consumir más alcohol cuando bebe & 25 & 2,5 & 18 & 3,5 & 46 & 3,5 \\
\hline \multicolumn{7}{|l|}{ Percepciones de suicidio y autoestima } \\
\hline Ha pensado en el suicidio pero no lo haría & 295 & 30,5 & 141 & 27,0 & 498 & 38,0 \\
\hline Lo ha pensado y le gustaría hacerlo (lo ha planeado) & 42 & 4,3 & 21 & 4,0 & 66 & 5,0 \\
\hline Ha buscado ayuda por haberlo pensado & 105 & 11,2 & 31 & 6,1 & 128 & 10,1 \\
\hline Cree que en futuro alcanzará lo que quiere & 718 & 74,3 & 386 & 73,9 & 1084 & 82,7 \\
\hline No cree que alcanzará lo que quiere & 34 & 3,5 & 18 & 3,4 & 48 & 3,7 \\
\hline No se imagina cómo será su futuro & 214 & 22,2 & 118 & 22,6 & 178 & 13,6 \\
\hline Está satisfecho con el aspecto y la forma de ser & 657 & 69,3 & 326 & 65,2 & 750 & 58,6 \\
\hline Más o menos satisfecho & 229 & 24,2 & 120 & 24,0 & 387 & 30,3 \\
\hline Poco o nada satisfecho & 62 & 6,5 & 54 & 10,8 & 142 & 11,1 \\
\hline Le gustaría cambiar muchas partes de su cuerpo & 38 & 4,0 & 24 & 4,8 & 73 & 5,7 \\
\hline Algunas partes & 160 & 16,8 & 71 & 14,1 & 226 & 17,6 \\
\hline Pocas partes & 232 & 24,4 & 101 & 20,0 & 272 & 21,2 \\
\hline No cambiaría nada & 520 & 54,7 & 309 & 61,2 & 710 & 55,4 \\
\hline \multicolumn{7}{|l|}{ Los compañeros dicen que usted es torpe } \\
\hline Muchos lo dicen & 18 & 1,9 & 19 & 3,8 & 48 & 3,8 \\
\hline Algunos lo dicen & 61 & 6,5 & 40 & 7,9 & 129 & 10,1 \\
\hline Muy pocos lo dicen & 177 & 18,7 & 129 & 25,6 & 346 & 27,1 \\
\hline Ninguno lo dice & 689 & 72,9 & 316 & 62,7 & 753 & 59,0 \\
\hline \multicolumn{7}{|l|}{ Riesgo de violencia } \\
\hline Ha sido golpeado en último año & 273 & 28,8 & 157 & 30,5 & 494 & 37,9 \\
\hline Golpeó a alguien el último año (pelea) & 304 & 32,1 & 171 & 33,6 & 478 & 36,7 \\
\hline Lleva un arma rutinariamente para defenderse & 25 & 2,6 & 18 & 3,5 & 38 & 2,9 \\
\hline Lleva un arma ocasionalmente para defenderse & 61 & 6,4 & 49 & 9,6 & 104 & 8,0 \\
\hline \multicolumn{7}{|l|}{ Protecciones } \\
\hline Practica su religión & 273 & 29,0 & 143 & 28,9 & 355 & 27,8 \\
\hline Se considera una persona religiosa & 535 & 57,0 & 288 & 57,9 & 782 & 61,2 \\
\hline
\end{tabular}

Las familias nuclares están disminuyendo mientras que las incompletas están aumentando. La violencia física se está acrecentando. 
Tabla 3. Odds ratios entre haber planeado versus no haber pensado en el suicidio y edad, sexo, estado de ánimo, función familiar, beber alcohol, percepciones de autoestima y de religiosidad de adolescentes de un colegio en tres momentos diferentes (2006, 2013 y 2018).

\begin{tabular}{|c|c|c|c|c|c|c|c|c|}
\hline \multirow{3}{*}{ VARIABLE } & \multicolumn{4}{|c|}{$\begin{array}{l}\text { PENSAR EN EL } \\
\text { SUICIDIO }\end{array}$} & \multirow{3}{*}{ OR } & \multirow{3}{*}{$\mathbf{L i}$} & \multirow{3}{*}{ Ls } & \multirow{3}{*}{$\begin{array}{l}\text { Valor } \\
\text { p }\end{array}$} \\
\hline & \multicolumn{2}{|c|}{$\begin{array}{c}\text { Lo ha } \\
\text { pensado y } \\
\text { lo ha } \\
\text { planeado }\end{array}$} & \multicolumn{2}{|c|}{$\begin{array}{l}\text { No lo ha } \\
\text { pensado }\end{array}$} & & & & \\
\hline & $\mathbf{N}$ & $\%$ & $\mathbf{N}$ & $\%$ & & & & \\
\hline $\begin{array}{l}\text { De } 17 \text { a } 20 \text { años } \\
\text { De } 10 \text { a } 16 \text { años }\end{array}$ & $\begin{array}{r}25 \\
103\end{array}$ & $\begin{array}{r}13 \\
6\end{array}$ & $\begin{array}{r}161 \\
1543\end{array}$ & $\begin{array}{l}87 \\
94\end{array}$ & 2,3 & 1,4 & 3,7 & 0,000 \\
\hline $\begin{array}{l}\text { Masculino } \\
\text { Femenino }\end{array}$ & $\begin{array}{l}53 \\
75\end{array}$ & $\begin{array}{l}5 \\
9\end{array}$ & $\begin{array}{l}955 \\
770\end{array}$ & $\begin{array}{l}95 \\
91\end{array}$ & 0,6 & 0,3 & 0,8 & 0,002 \\
\hline $\begin{array}{l}\text { Triste } \\
\text { Feliz }\end{array}$ & $\begin{array}{l}41 \\
26\end{array}$ & $\begin{array}{r}44 \\
4\end{array}$ & $\begin{array}{r}53 \\
584\end{array}$ & $\begin{array}{l}56 \\
96\end{array}$ & 17 & 9 & 30 & 0,000 \\
\hline $\begin{array}{l}\text { Disfunción severa } \\
\text { Funcional }\end{array}$ & $\begin{array}{l}24 \\
51\end{array}$ & $\begin{array}{r}38 \\
4\end{array}$ & $\begin{array}{r}39 \\
1248\end{array}$ & $\begin{array}{l}62 \\
96\end{array}$ & 15 & 8,4 & 26,9 & 0,000 \\
\hline $\begin{array}{l}\text { Ha bebido alcohol en la última semana } \\
\text { No bebió alcohol en la última semana }\end{array}$ & $\begin{array}{l}54 \\
75\end{array}$ & $\begin{array}{r}10 \\
6\end{array}$ & $\begin{array}{r}471 \\
1240\end{array}$ & $\begin{array}{l}90 \\
96\end{array}$ & 1,8 & 1,3 & 2,7 & 0,001 \\
\hline $\begin{array}{l}\text { Necesita consumir más alcohol cuando bebe } \\
\text { No necesita consumir más alcohol cuando bebe }\end{array}$ & $\begin{array}{r}18 \\
111\end{array}$ & $\begin{array}{r}34 \\
6\end{array}$ & $\begin{array}{r}35 \\
1671\end{array}$ & $\begin{array}{l}66 \\
94\end{array}$ & 7,7 & 4,2 & 14 & 0,000 \\
\hline $\begin{array}{l}\text { No cree que alcanzará lo que quiere } \\
\text { Cree que en el futuro alcanzará lo que quiere }\end{array}$ & $\begin{array}{l}21 \\
78\end{array}$ & $\begin{array}{r}36 \\
5\end{array}$ & $\begin{array}{r}38 \\
1356\end{array}$ & $\begin{array}{l}64 \\
95\end{array}$ & 9,6 & 5,3 & 17,1 & 0,000 \\
\hline $\begin{array}{l}\text { Poco o nada satisfecho } \\
\text { Satisfecho con su aspecto y forma de ser }\end{array}$ & $\begin{array}{l}43 \\
47\end{array}$ & $\begin{array}{r}30 \\
4\end{array}$ & $\begin{array}{r}98 \\
1186\end{array}$ & $\begin{array}{l}70 \\
96\end{array}$ & 11 & 6,9 & 17,5 & 0,000 \\
\hline $\begin{array}{l}\text { Ha sido golpeado en el último año } \\
\text { No ha sido golpeado en el último año }\end{array}$ & $\begin{array}{l}59 \\
81\end{array}$ & $\begin{array}{r}12 \\
6\end{array}$ & $\begin{array}{r}446 \\
1255\end{array}$ & $\begin{array}{l}88 \\
96\end{array}$ & 2 & 1,4 & 2,9 & 0,000 \\
\hline $\begin{array}{l}\text { No se considera una persona religiosa } \\
\text { Se considera una persona religiosa }\end{array}$ & $\begin{array}{l}69 \\
53\end{array}$ & $\begin{array}{r}10 \\
5\end{array}$ & $\begin{array}{r}613 \\
1022\end{array}$ & $\begin{array}{l}90 \\
95\end{array}$ & 2,1 & 1,4 & 3,1 & 0,000 \\
\hline No práctica su religión & 85 & 7 & 1142 & 93 & & & & \\
\hline Practica su religión & 34 & 6 & 498 & 94 & 1 & 0,7 & 1,6 & 0,600 \\
\hline
\end{tabular}

\section{DISCUSIÓN}

En este estudio existe posibilidad de sesgo dado que los adolescentes encuestados pudieron no haber respondido con total honestidad, lo cual trató de mitigarse realizando una encuesta de carácter voluntaria y anónima; además una de las limitaciones principales corresponde a la comprensión de las preguntas y respuestas dentro de los grupos de edad menores, por lo cual los investigadores acompañaron el proceso para la resolución de dudas.

El estado de ánimo triste es la principal relación estadística hallada en torno a la planeación suicida (tabla 3). González (24) ya ha planteado que la percepción del ánimo, si bien puede cambiar con base en lo que suceda, la tendencia es a permanecer o regresar al mismo estado luego del cambio momentáneo (tabla 1), por lo cual y dado su mínima variación a lo largo de los años, puede servir de forma indirecta para identificar adolescentes con trastornos del ánimo y con riesgo de suicidio.

Lo propio puede afirmarse sobre la percepción de la funcionalidad familiar. La tabla 2 pone de manifiesto, particularmente en el caso de la disfunción familiar severa $(20,6)$, lo poco que cambia tal percepción. Dada su relación con la planeación suicida (tabla 3 ) se propone que también sirva como un indicador de ideación suicida a tener en cuenta.
En la tabla 2 queda expuesto cómo el consumo de alcohol es más o menos estable a lo largo de los años (poco más de 1 de 4 jóvenes han bebido en la última semana) y cómo entre un $7 \%$ y un $2 \%$ de los jóvenes ya presentan un consumo patológico (de acuerdo al test de Cage, con una respuesta positiva de cualquiera de las cuatro que lo constituyen). La tabla 2, asimismo, muestra, y confirma, la relación entre consumo patológico e ideas suicidas (tabla 3), asociación bien estudiada (16).

También llama la atención la frecuencia más o menos estable a lo largo de los años de entre un $4 \%$ y un $5 \%$ de adolescentes que han presentado ideación suicida (piensa y les gustaría suicidarse). ¿Serán jóvenes con trastornos depresivos patológicos, tal y como menciona el estudio de Salud Mental de 2015? (27).

No sorprende la asociación entre las percepciones de baja autoestima y la planeación suicida (tabla 3 ). Incluso tales percepciones (7), podrían servir como orientadores para identificar población que requiera un programa de prevención del suicidio.

La tabla 3 confirma la relación entre violencia e ideas suicidas, aunque se suponía una asociación más poderosa. Asimismo, la relación entre religiosidad medida con las preguntas elaboradas no fue muy fuerte (tabla 3 ). 


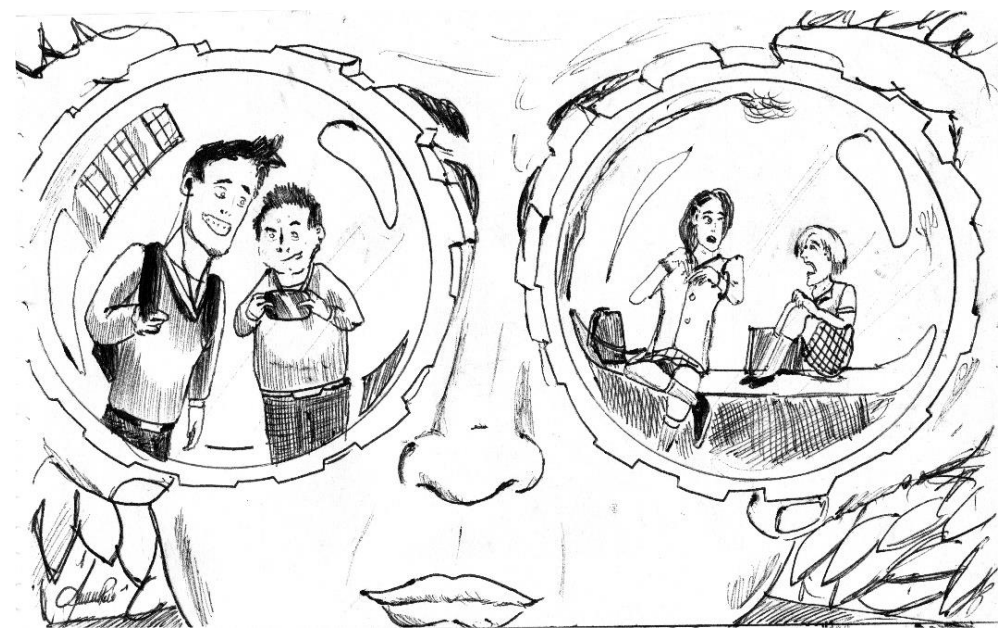

En conclusión, para responder a la orientadora del colegio mencionada al principio y partiendo de que el Departamento de Medicina Comunitaria de la FUJNC viene construyendo proyectos de consejería en tal institución, se propone que, a través del instrumento para determinar riesgos psicosociales que se aplica cada cierto tiempo, se identifique como población de riesgo para suicidio a aquellos adolescentes que persistentemente presenten una percepción de ánimo con tendencia a la tristeza, problemas severos en sus relaciones familiares, consumo problemático de alcohol y que no se perciban bien a sí mismos. Con base en los resultados de estudios como este, se puede invitar a esta población a asistir a consejerías (28) donde se aborden estos aspectos (29).

Ratifica esta investigación el probable potencial que puede alcanzarse por unir colegios con facultades del área de la salud.

\section{REFERENCIAS}

(1) Ministerio de Salud y Protección Social y Colciencias [Internet]. Encuesta Nacional de Salud Mental 2015 [citado 21 oct 2018]. Disponible en: https://goo.gl/sWdvSi

(2) Who.int [Internet]. Organización Mundial de la Salud; 2018 [actualizado 24 Ago 2018; citado 21 oct 2018]. Disponible en: https://goo.gl/k8jKiL

(3) Unicef.org [Internet]. Suicidio. Comunicación, infancia y adolescencia. Guía para periodistas; 2017 [actualizado 2017; citado 21 oct 2018]. Disponible en: https://goo.gl/gxzLzS

(4) Franco SA, Gutiérrez ML, Sarmiento J, Cuspoca D, Tatis J, Castillejo A Suicide in University Students in Bogotá, Colombia, 2004-2014. Cien Saude Colet. 2017 Jan;22(1):269-278. Disponible en: https://bit.ly/2RyKOxH

(5) Alonso LM, Murcia G, Murcia J, Herrera D, Gómez D, Comas M et al. Autoestima y relaciones interpersonales en jóvenes estudiantes de primer semestre de la División Salud de la Universidad del Norte, Barranquilla (Colombia). Salud Uninorte. Barranquilla (Col.) 2007 23(1):32-42. Disponible en: https://bit.ly/2poE7NP

(6) Monge JA, Cubillas MJ, Roman R, Abril E. Intentos de suicidio en adolescentes de educación media superior y su relación con la familia. Psicología y salud $2007 ; 17(1): 45-51$. Disponible en: https://bit.ly/2FBcHhi

(7) Sánchez JC, Musitu G, Villareal ME, Martínez B. Ideación suicida en adolescentes: Un análisis psicosocial. Colegio Oficial de Psicólogos de Madrid.2012; 19(3):279-287. Disponible en: https://bit.ly/2NX6At1

(8) Rodríguez C, Caño A. Autoestima en la adolescencia: Análisis y estrategia de intervención. International Journal of Psychology and Psychological Therapy.2012; 12(3):389-403 Disponible en: https://bit.ly/2FBZLHK

(9) Zimmerman MA, Copeland LA, Shope JT, Dielma, TE. A Longitudinal Study of Self-Esteem: Implications for Adolescent Development. Journal of Youth and Adolescence.1997;26(2): 117-141. Disponible en: https://bit.ly/2SWn6bc

(10) Who.int [Internet]. Organización Mundial de la Salud; 2005. Disponible en: https://bit.ly/2iN8Ks0

(11) Brunstein KA, Marrocco F, Kleinman M, Schonfeld IS, Gould MS. Bullying, Depression, and Suicidality in Adolescents. J Am Acad Child Adolesc Psychiatry. 2007; 46(1): 40-49.

(12) Albores L, Sauceda JM, Ruiz S, Roque E. El acoso escolar (bullying) y su asociación con trastornos psiquiátricos en una muestra de escolares en México. Salud pública Méx [Internet]. 2011 Jun [citado 2018 Oct 21]; 53(3): 220-227. Disponible en: https://bit.ly/2DbhCUz.

(13) Arroyave P. Factores de vulnerabilidad y riesgo asociados al bullying. revista CES Psicología [Internet]. 2012 [citado 21 octubre 2018];5(1):116-125. Disponible en: https://bit.ly/2MgzwZ2

(14) DANE (9 de mayo de 2012). Muestreo: Situación de los colegios en Bogotá es preocupante: DANE. www.elespectador.com publicación online. Disponible en: https://bit.ly/2FCnwQ3

(15) Instituto Nacional de Medicina Legal y Ciencias Forenses. Centro de Referencia Nacional sobre Violencia (2011). Forensis 2016. Datos para la vida [citado 4 de noviembre 2018]. Disponible en: https://bit.ly/2t4H9vI
(16) Evans E, Hawton K, Rodham K. Factors Associated with Suicidal Phenomena in Adolescents: A Systematic Review of Population-based Studies. Clin Psychol Rev. 2004; 24(8): 957-79.

(17) Médicos y pacientes.com. (2018). El alcohol y la depresión, factores de riesgo de suicidio más prevalentes en pacientes con patología dual [Internet]. Médicos y Pacientes [citado 22 octubre 2018]. Disponible en: https://bit.ly/1qDHWwW

(18) Artículo 16.3. Declaración Universal de los Derechos Humanos. Asamblea General de las Naciones Unidas (1948). La familia es el elemento natural y fundamental de la sociedad y tiene derecho a la protección de la sociedad y del Estado.

(19) Bezanilla JM, Miranda A. La familia como grupo social: una reconceptualización. Alternativas en psicología. 2013; 17(29):58-73. Disponible en: https://bit.ly/2CmkJY6

(20) Cogollo Z, Gómez E, De Arco C, Ruíz I, Campo A. Asociación entre disfuncionalidad familiar y síntomas depresivos con importancia clínica en estudiantes de Cartagena, Colombia. Rev. Colomb. Psiquiat.2009. 38(4):637-644.

(21) McArdle P, Wiergersma A, Gilvarry E, Kolte B, McCarthy S, Fitzgerald M, et al. European Adolescent Substance Use: The Roles of Family Structure Function and Gender. Addiction. 2002;97(3):329-36.

(22) Engels RC, Vermulst AD, Dubas JS, Bot SM, Gerris J. Long-term Effects of Family Functioning and Child Characteristics on Problem Drinking in Young Adulthood. Eur Addict Res. 2005;11(1):32-7.

(23) Bernt F. Una fe que haga justicia: Orientaciones religiosas y sus correlatos sociales [citado 17 enero 2019]. Ciencias Psicológicas. 2015. 1(2):171-178. Disponible en: https://revistas.ucu.edu.uy/index.php/cienciaspsicologicas/article/view/5 68

(24) Dyche L, Zayas LH. The value of Curiosity and Naiveté for the Crosscultural Psychotherapist. Fam Process 1995; 34: pp. 389-399.

(25) Smilkstein G. The family APGAR: A Proposal for a Family Function Test and its Use by Physicians. J Fam Pract. 1978 Jun;6(6):1231-9.

(26) Ewing JA. Detecting Alcoholism. The CAGE Questionnaire. JAMA. 1984;252:1911-1912

(27) González JC, De La Hoz F. Relaciones entre los comportamientos de riesgo psicosociales y la familia en adolescentes de Suba, Bogotá. Revista de Salud Pública. 2011;13(1):67-78 [citado 5 enero 2019]. Disponible en: https://bit.ly/2TPH1IT

(28) Ministerio de Protección Social. Encuesta Nacional de Salud Mental 2015. Disponible en: https://bit.ly/1KHISm7

(29) OMS. Skills for Health Skills-based Health Education Including Life Skills An Important Component of a Child-Friendly/Health-Promoting School [Internet]. Document 9 Information Series On School Health. 2001. Disponible en: https://goo.gl/LfHvvj 


\section{Índice de las investigaciones publicadas de 2015 a 2019} Título Autor

\begin{tabular}{|c|c|c|c|}
\hline Título & Autor & Número & Enlace \\
\hline $\begin{array}{l}\text { Estudio microbiológico del reuso y esterilización } \\
\text { de limas endodónticas como práctica segura }\end{array}$ & $\begin{array}{l}\text { Ruth Yamile Gómez, } \\
\text { Diana Marcela Rivera G. }\end{array}$ & $\begin{array}{l}\text { Vol. 23, } \\
\text { Núm. 132 } \\
\text { (2015) }\end{array}$ & https://goo.gl/Wyr6sc \\
\hline $\begin{array}{l}\text { ¿Qué está ocurriendo con el estado de salud, en cuanto a } \\
\text { morbilidad, de } 380 \text { adultos mayores de } 60 \text { años de la localidad } \\
\text { de Suba de la ciudad de Bogotá desde el } 2014 \text { al 2015? }\end{array}$ & $\begin{array}{l}\text { Rosa Isabel Castro C., } \\
\text { Natalia Gómez A. }\end{array}$ & $\begin{array}{l}\text { Vol. 23, } \\
\text { Núm. } 133 \\
(2015)\end{array}$ & https://goo.gl/YEcGck \\
\hline $\begin{array}{c}\text { Satisfacción de pacientes que asisten a consulta médica } \\
\text { ambulatoria en una EPS de régimen especial }\end{array}$ & $\begin{array}{l}\text { Juan Carlos González Q., Sergio Díaz, } \\
\text { Stephanie Hernández, Steven } \\
\text { Huérfano, Stefanía Ibarra, } \\
\text { Alejandra Jiménez, Diana Lara, } \\
\text { Isabel León, Estefany Lizcano. }\end{array}$ & $\begin{array}{l}\text { Vol. 23, } \\
\text { Núm. 134 } \\
\text { (2015) }\end{array}$ & https://goo.gl/mm1rhm \\
\hline $\begin{array}{c}\text { Investigación formativa en Medicina Comunitaria } \\
\text { en } 2015\end{array}$ & Juan Carlos González Q. & \multirow[b]{2}{*}{$\begin{array}{l}\text { Vol. 23, } \\
\text { Núm. 135 } \\
(2015)\end{array}$} & https://goo.gl/DBgorC \\
\hline $\begin{array}{l}\text { Cambios en el consumo de alcohol en adolescentes } \\
\text { escolarizados de la localidad de Suba entre los años } \\
\qquad 2010 \text { y } 2015\end{array}$ & $\begin{array}{l}\text { Juan Carlos González Q., Andrés Felipe } \\
\text { Blanco H., Juan Camilo Borrás A., } \\
\text { Johandra Viviana Camacho L., Karen } \\
\text { Andrea Camacho S., Diego Alexander } \\
\text { Camargo V. }\end{array}$ & & https://goo.ql/zY2V1W \\
\hline $\begin{array}{l}\text { Percepción del proceso de quejas y reclamos } \\
\text { en una facultad de Medicina }\end{array}$ & $\begin{array}{c}\text { Juan Carlos González, María Montaña, } \\
\text { Juan Morales, Erika Moreno, Ada } \\
\text { Olmos, Laura Ortiz, Juanita Ovalle, } \\
\text { Diana Ramírez. }\end{array}$ & \multirow{2}{*}{$\begin{array}{l}\text { Vol. 24, } \\
\text { Núm. 137 } \\
\text { (2016) }\end{array}$} & https://goo.gl/Wq9z8N \\
\hline $\begin{array}{l}\text { Descripción de la participación estudiantil } \\
\text { en una facultad de Medicina de } 2010 \text { a } 2013\end{array}$ & $\begin{array}{l}\text { Juan Carlos González, Laura Masmela, } \\
\text { Daniel Mesa, Jessica Mora, Laura } \\
\text { Murillo, Mitndbaim Parra, Natalia Pérez, } \\
\text { Ximena Pinedo, Maurent Prieto, } \\
\text { Angélica Quintero. }\end{array}$ & & https://goo.gl/uuTRBD \\
\hline $\begin{array}{l}\text { Influencia del inicio de relaciones sexuales } \\
\text { en comportamientos violentos en adolescentes } \\
\text { de colegios públicos de la localidad de Suba }\end{array}$ & $\begin{array}{l}\text { Juan Carlos González Q., Carolina } \\
\text { Mendoza V., Angie Stephanny Mojica } \\
\text { R., Ana Fernanda Nicola Y. }\end{array}$ & $\begin{array}{l}\text { Vol. 24, } \\
\text { Núm. } 138 \\
(2016)\end{array}$ & https://goo.gl/58NsrC \\
\hline $\begin{array}{l}\text { Relación entre violencia, consumo de alcohol y tabaco } \\
\text { en adolescentes escolarizados en Suba en } 2010\end{array}$ & $\begin{array}{l}\text { Juan Carlos González Q., María } \\
\text { Alejandra Miranda M., Angie Lizeth } \\
\text { Monroy M., Liliana Andrea Naranjo A. }\end{array}$ & $\begin{array}{l}\text { Vol. 24, } \\
\text { Núm. } 139 \\
(2016)\end{array}$ & https://goo.gl/spRTXA \\
\hline $\begin{array}{l}\text { Diferencia de comportamientos violentos } \\
\text { entre un colegio de estratos } 4 \text { y } 5 \text { y uno de estrato dos, Suba } \\
\text { (Bogotá) }\end{array}$ & $\begin{array}{c}\text { Jesús David Achury G., Marialy Ariza } \\
\text { R., Luisa Fernanda Arrieta U., María } \\
\text { Camila Balanta M., Juan Carlos } \\
\text { González Q. }\end{array}$ & \multirow{2}{*}{$\begin{array}{l}\text { Vol. 24, } \\
\text { Núm. } 140 \\
(2016)\end{array}$} & https://goo.gl/5qhV4D \\
\hline $\begin{array}{l}\text { Entre el idealismo mágico y el racionalismo positivista. } \\
\text { Sobre la eficacia sanadora de las cadenas de oración }\end{array}$ & Jairo Echeverry R. & & https://goo.gl/tXqLEA \\
\hline $\begin{array}{c}\text { Diferencias de riesgos psicosociales entre dos colegios, } \\
\text { uno estrato bajo y otro estrato alto }\end{array}$ & Juan Carlos González Q. & $\begin{array}{l}\text { Vol. 24, } \\
\text { Núm. } 141 \\
(2016)\end{array}$ & https://goo.gl/US1g9B \\
\hline $\begin{array}{l}\text { Relación entre trastorno de conducta alimentaria } \\
\text { y género y familia en adolescentes escolarizados, } \\
\text { Suba (Bogotá) }\end{array}$ & $\begin{array}{l}\text { Juan Carlos González Q., Diana } \\
\text { Carolina Martínez C., Sonia Gisela } \\
\text { Martínez M, Jenny Pinzón. }\end{array}$ & $\begin{array}{l}\text { Vol. 25, } \\
\text { Núm. } 143 \\
(2017)\end{array}$ & https://goo.gl/xDD1ke \\
\hline $\begin{array}{l}\text { Relación entre comportamientos de riesgo } \\
\text { en sexualidad y familia en adolescentes escolarizados, } \\
\text { 2015-2016 }\end{array}$ & $\begin{array}{c}\text { Juan Carlos González Q., Luisa } \\
\text { Fernanda Molano, Natalia Carolina } \\
\text { Montes, Daniela Alejandra Moreno, } \\
\text { Laura Camila Nova, Erika Daniela } \\
\text { Olaya, Jenny Carolina Páez, Jenny } \\
\text { Pinzón. }\end{array}$ & $\begin{array}{l}\text { Vol. 25, } \\
\text { Núm. 144 } \\
\text { (2017) }\end{array}$ & https://qoo.gl/U95a9S \\
\hline $\begin{array}{c}\text { Relación entre violencia, género y familia } \\
\text { en adolescentes de instituciones educativas distritales } \\
\text { de Suba, Bogotá }\end{array}$ & $\begin{array}{l}\text { Juan Carlos González Q., Nicolás } \\
\text { Méndez V., Ana María Morales G., } \\
\text { Angie Paola Murillo R., María Mercedes } \\
\text { Pallares V., Juan Diego Paloma M., } \\
\text { Jenny Pinzón. }\end{array}$ & $\begin{array}{l}\text { Vol. 25, } \\
\text { Núm. } 145 \\
\text { (2017) }\end{array}$ & https://goo.ql/5hBUA9 \\
\hline $\begin{array}{c}\text { Determinación de los comportamientos de sueño } \\
\text { en una muestra por conveniencia de estudiantes } \\
\text { de Medicina }\end{array}$ & $\begin{array}{l}\text { Juan Carlos González Q., Libi Leonor } \\
\text { Niño M., Jennifer Lissetd Pachón A., } \\
\text { Cambell Johanna Perea, Jenny Pinzón. }\end{array}$ & $\begin{array}{l}\text { Vol. 26, } \\
\text { Núm. 146 } \\
(2018)\end{array}$ & https://goo.gl/EbuRcG \\
\hline $\begin{array}{c}\text { Relación entre género y conocimientos, actitudes } \\
\text { y prácticas respecto al uso del condón en adolescentes } \\
\text { escolarizados, Suba (Bogotá), } 2016\end{array}$ & $\begin{array}{l}\text { Juan Carlos González Q., Daniela } \\
\text { Katherin Morales D., Juan José Murcia } \\
\text { G., Natalia Muñoz B., Daniela Ospina } \\
\text { G., Laura Catalina Nossa C. }\end{array}$ & $\begin{array}{l}\text { Vol. 26, } \\
\text { Núm. 147 } \\
\text { (2018) }\end{array}$ & https://goo.gl/f4A8T] \\
\hline $\begin{array}{c}\text { Prevalencia de trastornos del sueño en niños } \\
\qquad y \text { adolescentes }\end{array}$ & $\begin{array}{l}\text { Juan Carlos González, Geraldinne } \\
\text { Acevedo, Paula Alarcón, Ángela Mileidy } \\
\text { Casas, Glendy Ardila, Julian Bernal, } \\
\text { Javier Avella, Jenny Pinzón. }\end{array}$ & $\begin{array}{l}\text { Vol. 26, } \\
\text { Núm. 148 } \\
\text { (2018) }\end{array}$ & https://goo.gl/4R2eLL \\
\hline $\begin{array}{c}\text { Relación entre el nivel nutricional y el índice de masa corporal } \\
\text { en población adolescente }\end{array}$ & $\begin{array}{l}\text { Andrea Caballero, María Casas, Laura } \\
\text { Camargo, Luz Betancur, Elizabeth } \\
\text { Jaramillo. Juan Carlos González, Jenny } \\
\text { Pinzón. }\end{array}$ & $\begin{array}{l}\text { Vol. 26, } \\
\text { Núm. 149 } \\
\text { (2018) }\end{array}$ & https://bit.ly/2GzGxmV \\
\hline $\begin{array}{l}\text { Factores asociados a ideas suicidas en adolescentes } \\
\text { escolarizados de Bogotá en 2006, } 2013 \text { y } 2018\end{array}$ & $\begin{array}{c}\text { Juan Carlos González, Catalina } \\
\text { Monroy., Laura Mogollón, Juan Molano, } \\
\text { Cristian Maturana, Nathalya Oñate, } \\
\text { María Ospina, Jenny Pinzón. } \\
\text { Dibujos: Andrés Reyes }\end{array}$ & $\begin{array}{l}\text { Vol. 27, } \\
\text { Núm. 150 } \\
\text { (2019) }\end{array}$ & Este número. \\
\hline
\end{tabular}

